\title{
3D Seismic Prestack viscoelastic migration method based on high-precision traveltime calculation in VTI medium
}

\author{
Qiannan Liu, Wenfeng Du, Jingtao Zhao,Xiaoting Li \\ China University of Mining \& Technology(Beijing) \\ State Key Laboratory of Coal Resources and Safe Mining \\ Beijing, China \\ lqn_cumtb@163.com
}

\begin{abstract}
In coal exploration, seismic strong reflections from overlying strata may shield the propagation of seismic waves into deep parts, resulting in poor imaging quality for deep targeted area. In fact, seismic wave energy will be lost and velocities will undergo dispersion after passing through viscoelastic medium. Therefore, a method is proposed for restoring the absorbed energy, namely $Q$ compensation. The process of absorption compensation can be decomposed into two parts, with one from zero offset and the other from finite offset. In order to improve the accuracy of absorption compensation for finite offset, we include sixth order term in polynomial expansion to form a high precision traveltime calculation formula in VTI medium. Field data application shows that the proposed prestack viscoelastic migration method can eliminate shielding effect and improve vertical resolution, which has important values in resolving the hidden seams.
\end{abstract}

Keywords-prestack migration; $Q$ compensation; traveltime calculation; VTI medium

\section{INTRODUCTION}

In the process of seismic wave propagation, the formation's viscoelastic characteristic will lead to the seismic wave energy attenuation ${ }^{[1][2]}$ and speed dispersion ${ }^{[3][4]}$. For coal exploration, a phenomenon is that the strong reflection effect of overlying strata generally impedes the propagation of seismic waves to deep parts, affecting exploration and development for targeted coal seam. Seismic migration methods are usually based on elastic frame, so they can't effectively solve such geological problems. As the real subsurface medium is not an ideal elastic body, it has obvious viscosity and a great absorption of seismic waves. Quality factor Q, as a significant characterization of stratum attenuation properties ${ }^{[5]}$ in oil \& gas prediction, is regarded as a prospective seismic technology.

Inverse $\mathrm{Q}$ filtering is a prevailing method for compensating absorption effect ${ }^{[6]}$. It can not only compensate amplitude attenuation and frequency loss, but also can improve the characteristic of recording phase. The method can improve the continuity of lineups and increase the energy of reflected wave, which will generate a good image with a higher SNR (signal to noise ratio) and resolution. At present, the inverse Q filtering method mainly has three methods: high frequency compensation in series expansion approximation method ${ }^{7][8]}$, the inverse $Q$ filtering method based on wave field continuation ${ }^{[9]}$ and other methods ${ }^{[10]}$.

In this work, we take the absorption compensation decomposition strategy, dividing compensation process into zero offset compensation and finite offset compensation ${ }^{[10]}$.This approach changes the conventional ideas of direct compensation, which considers absorption compensation from zero offset and from finite offset separately. Thus the accuracy of the $\mathrm{Q}$ compensation can be greatly improved if we can increase the accuracy of travltime calculation for finite offset. A higher order polygonal expansion for traveltime calculation in VTI medium is suggested for 3D seismic prestack migration in viscoelastic medium. Based on the high precision calculation, the accuracy of migration image will be improved. Therefore, this method has a good effect in eliminating the shielding effect from strong reflections.

\section{THEORY AND METHOD}

\section{A. The Physical Meaning of Quality Factor $Q$}

With seismic wave propagation in the medium, the elastic energy produced by the wave will gradually be absorbed by medium and finally converted into heat energy. The process of elastic energy converting into heat energy is called absorption ${ }^{[11]}$.Quality factor Q explains the proportion of energy loss, which is defined by the ratio of the original seismic wave energy to the energy dissipation within the scope of an seismic wavelength $\lambda^{[12]}$.

$$
Q=2 \pi \frac{E}{\Delta E}
$$

\section{B. Kjartansson Model}

According to the average storage energy $\omega$ and energy dissipation $\omega$ of a cycle sine deformation in viscoelastic medium, Q'connell and Budiansky propose a definition, namely 


$$
Q=\frac{4 \pi \omega}{\Delta \omega}
$$

where the factor $\mathrm{Q}$ in this definition is associated with phase angle 错误! 未找到引用源。 of stress and strain. The relationship is listed as follows

$$
\frac{1}{Q}=\tan \delta
$$

According to this formula, Kjartansson gives the frequency response of viscoelastic medium ${ }^{[13]}$. There is no cut-off frequency, and the response is completely determined by phase velocity under some reference frequency and $\mathrm{Q}$ value. Based on this model and considering the condition of weak absorption, it can approximately has

$$
Q(\omega) \approx \frac{1}{2 \tan \frac{\pi \gamma}{2}} \approx \frac{1}{\pi \gamma}
$$

\section{Compensation Decomposition}

The Q compensation is used for compensating absorbed seismic wave energy, namely inverse $Q$ filtering. The inverse Q filtering performs energy compensation mainly along the time direction, and does not apply to the wave field extrapolation of the migration direction. Therefore, it is needed to use a suitable method to compensate the energy absorption produced by finite offset.

Based on a fact that seismic wave energy compensation process can be divided into zero offset compensation and finite offset compensation, this paper improves the compensation part in finite offset by adopting a higher accuracy travletime calculation in VTI medium.

Based on the seismic wave transmission in viscoelastic medium, we can express the plane wave propagation equation as follows

$$
U(t, f)=U_{0}(f) \exp (-i 2 \pi f t) \exp \left(-\frac{\pi f t}{Q}\right) \exp \left(\frac{i 2 f t}{Q} \ln \left|\frac{f}{f_{r}}\right|\right)
$$

Define $g(t, f)=\exp (c(f) \alpha t) c(f)=-\pi f+i 2 f \ln \left|\frac{f}{f_{r}}\right|$ Break $g(t, f)$ into two parts,namely

$$
g(t, f)=g_{0}\left(t_{0}, f\right) g_{x}(x, f)
$$

Among them, $g_{0}\left(t_{0}, f\right)$ stands for zero offset effect, $g_{x}(x, f)$ stands for finite offset effect .Thus, we can get the basic formula of inverse $\mathrm{Q}$ filter output

$$
y(t)=\int x(\tau) g(\tau, t) d \tau
$$

The calculation of $g(\tau, t)$ can be obtained by solving the matrix equation $G x=y$, which can be solved by regularized least squares [10].After estimated the $\mathrm{Q}$ value and calculated the $\mathrm{G}$ matrix, we can get the output $\mathrm{y}(\mathrm{t})$ about the offset $\mathrm{Q}$ compensation.

\section{Traveltime Calculation in VTI Medium}

In VTI medium, the traveltime of seismic wave can be expressed in the following formulas

$$
\begin{gathered}
t(p)=\int \frac{d z}{v^{2} \sqrt{1-v^{2} p^{2}}}=\int \frac{d t}{v \sqrt{1-v^{2} p^{2}}} \\
x(p)=\int \frac{p}{q} d z=\int \frac{v p}{\sqrt{1-v^{2} p^{2}}} d z=\int \frac{v^{2} p}{\sqrt{1-v^{2} p^{2}}} d t
\end{gathered}
$$

where $p=\frac{d t}{d x}$.

To adapt for the situation of larger offset, we apply a high order expansion as follows

$$
\begin{gathered}
x(p)=p t_{0} v_{2}^{2}+\frac{1}{2} p^{3} t_{0} v_{4}^{4}+\frac{3}{8} p^{5} t_{0} v_{6}^{6}+o\left(p^{7}\right) \\
t(p)=t_{0}+\frac{1}{2} p^{2} t_{0} v_{2}^{2}+\frac{3}{8} p^{4} t_{0} v_{4}^{4}+\frac{15}{48} p^{6} t_{0} v_{6}^{6}+o\left(p^{7}\right)
\end{gathered}
$$

Parameter $p$ can be obtained by giving initial ray parameters $p_{0}$ through quasi-newton method.

Using a higher order polynomial expansion, we can increase the accuracy of traveltime calculation. Therefore, the corresponding migration imaging quality can be improved in viscoelastic medium.

\section{FIELD EXAMPLE}

In mine exploration, overlying strata with a strong wave impedance brings great challenges to seismic exploration, which will produce shielding effect hindering seismic energy propagation to deep targeted areas. In order to effectively solve this problem, we present 3D prestack viscoelastic migration strategy, with the purpose for compensating absorbed energy. The absorption energy are decomposed into two parts, with one from zero offset and the other from finite offset.

In 3D prestack migration process, a high precision traveltime calculation formula in VTI medium is expanded to sixth order to meet the compensation for large offsets. We also optimized migration parameters to obtain a good imaging result, with migration aperture equal to 2000 meters and the maximum migration dip constrained to 60 degree for antialiasing.

One inline direction profile from conventional 3D prestack time migration is shown in Figure 1 and the corresponding 3D viscoelastic prestack time migration profile is shown in Figure 2. Comparing the marked areas with red circles, we can clearly see the improved image quality, with more continuous seismic events and higher vertical resolution.

The crossline direction profile can also view dramatic image improvement, as marked with red squares in conventional 3D prestack time migration Fig.3 and 3D viscoelastic prestack time migration profile Fig.4, respectively. Field application demonstrates the imaging improvements for the targeted layers and also verifies the effectiveness of the 
proposed method in solving shielding problems from strong reflections.

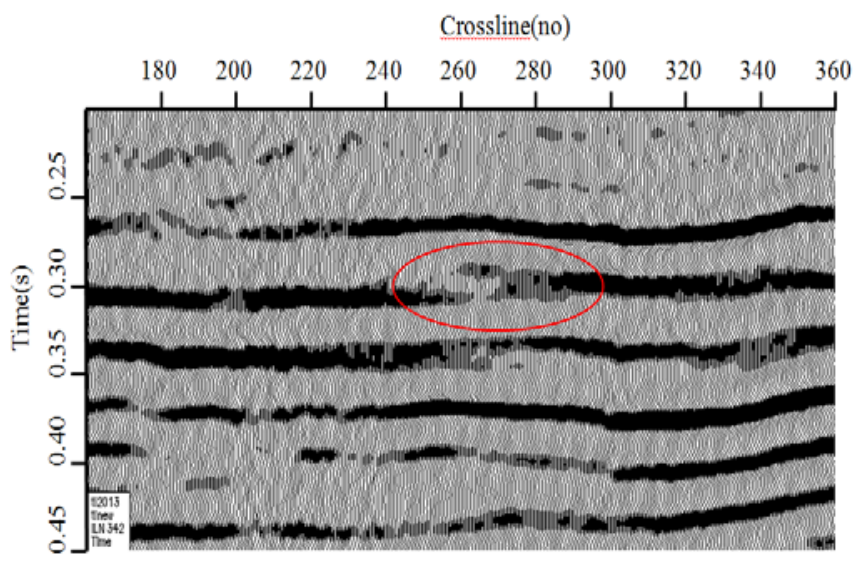

Fig. 1. Conventional 3D prestack time migration profile in inline direction

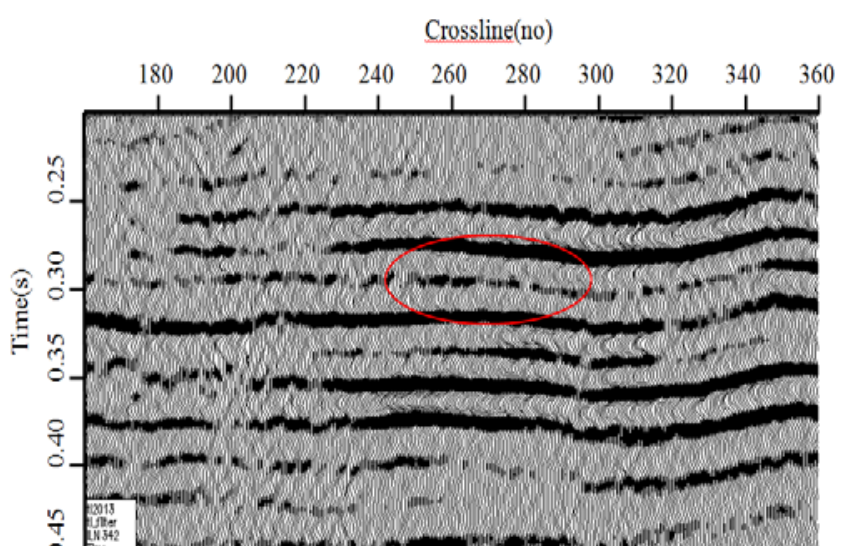

Fig. 2. 3D seismic prestack viscoelastic time migration profile in inline direction

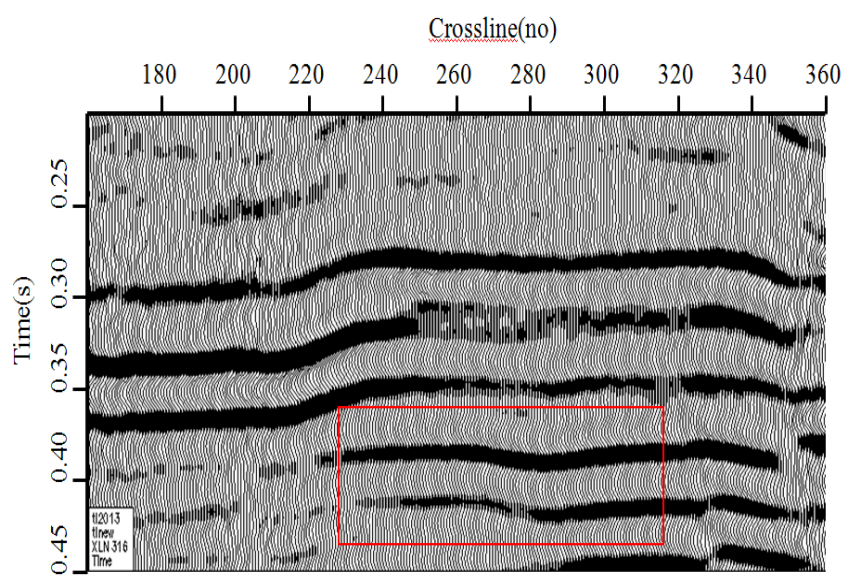

Fig.3. Conventional 3D prestack time migration profile in crossline direction

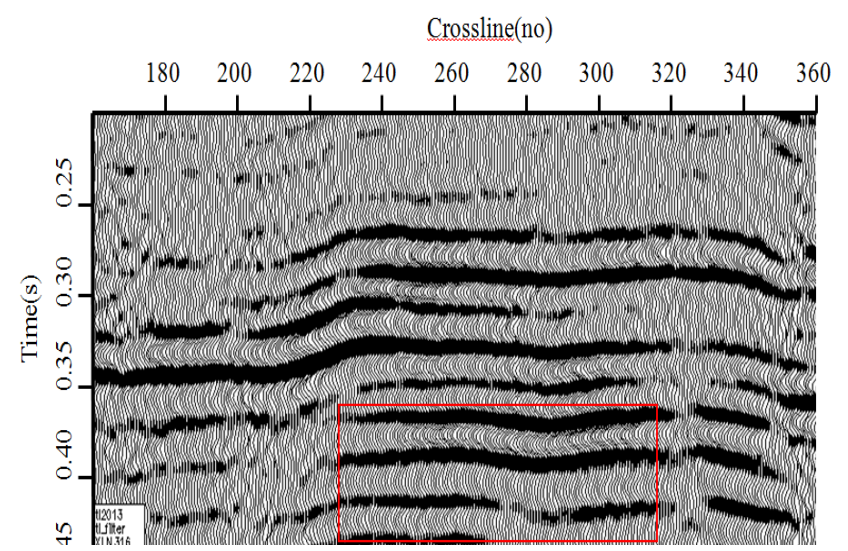

Fig.4. 3D seismic prestack viscoelastic time migration profile in crossline direction

\section{CONCLUSION}

Through compensation for energy attenuation and velocity dispersion in migration process, we develop a 3D seismic viscoelastic prestack migration method, which considers the compensation from zero offset and finite offset, respectively. In order to better calculate the traveltime for long offsets, a high precision traveltime calculation formula in VTI medium is proposed. Field application verifies the effectiveness of the method in eliminating shielding effect from strong reflections of overlying strata and its important values for clarifying hidden seams. Although, there are many views on the viscoelastic model building, how to optimize an appropriate viscoelastic model according to specific geological conditions is an open issue.

\section{ACKNOWLEDGMENT}

This research is supported by the National Science and Technology Supporting Program (2012BAB13B01), National Key Scientific Instrument and Equipment Development Program (2012YQ030126), Coal United Project of National Natural Science Foundation (U1261203).We also express great appreciation for the anonymous reviewers and editors.

\section{REFERENCES}

[1] Hamilton E L.Compressional-wave attenuation in marine sediments.Geophysics,1972,37(4),pp.620-646.

[2] Toksoz M N,Johnston D H,Timur A.Attenuation of seismic waves in dry and saturated rocks:1.Laboratory measurements.Geophysics, 44(4), pp.681-690.

[3] Sams,M S ,Neep,J P,Worthington,M H,King,M S.The measurement of velocity in sedimentary rocks.Geophysics,1997,62(5),pp.1456-1464.

[4] Spencer T W,Sonnadt J R,Butlers T M.Seismic Q stratigraphy or dissipation.Geophysics,1982,47(1),pp.16-24.

[5] Wu L, Zhang G Z. Quality factor calculate and compensation method [D].China petroleum university, 2011.

[6] Yu Z, Wang Y C, He J,Yang R. Inverse Q filtering method [J]. Progress in exploration geophysics, 2009, 32 (5) ,pp.309-314.

[7] Hargreaves,N.D,A.J.Calvert.Inverse $Q$ filtering by Fourier transform[J].Geophysics,56,pp.519-527.

[8] Bickel S H,Natarajan R R.Plane-wave Q deconvo -lution[J].Geophysics 1985,50(9),pp.1426-1439. 
[9] Wang $\mathrm{Y}$ H.Inverse Q-filter for seismic resolution enhancement [J].Geophysics,2006,71(3),pp.V51-V60.

[10] Li G F,Liu Y,Zheng H,Huang W.Absorption decomposition and compensation via a two-step scheme[J].Geophysics, VOL.80, NO.6, pp.V145-V155.

[11] Yao Y. The seismic wave field and seismic exploration [M]. Geological publishing house, 2006,pp.145-150.
[12] Li Z C, Zhang J H. Seismic data processing method [M]. China university of petroleum press, 2004, 8,pp.109-113.

[13] Pei J Y, He Q. Based on the inverse Q filtering Kjartansson model [J]. Progress in geophysics, 1994, 9 (1) ,pp.90-97. 